Tabanus fumidus Austen. Tabanidæ. Mésopotamie. Ibid., p. 281.

Tabanus inaequatus Austen Tabanida. Mésopotamie. Ibid., p. 284.

Tabanus sp. incert. nov? Austen. Tabanidæ. Bagdad, Mésopotamie. Ibid., p. 289.

Tabanus sp. incert. (voisin de $T$. sufis Jeannike) Austen. Tabanidæ. Fao, golfe Persique. Ibid., p. 290.

Simulium bipunctatum Austen. Simulidæ. Mésopotamie. Ibid., p. 275.

Phlebotomus Annandalei Sinton. Psychodida. Madras. Indian Journ. med. research, X, janvier 1923 , p. 742.

Stegomyia Edwardsi Barraud. Culicidr. Iles Andaman. Ibid., p. 784.

Christophersomyia Barraud. Culicidæ. Créé pour Stegomyia Thomsoni Theobald, 1905, et St. annulirostris Theobald, 1905 de l'Inde anglaise. Indian Journ. med. research, X, janvier 1923, p. 786.

Tabanus cordigeroides Surcouf. Tabanidæ. Djamâa, sud constantinois, Algérie. Ann. Soc. Entomologique de France, XCI, 1922 (mars 1923), p. 237.

Atylotus Guyonae Surcouf. Tabanidx. El Golea, département d'Alger. Ibid., p. 238.

Ochrops Kroberi Surcouf. Tabanida. Ourir, sud constantinois. Ibid., p. 239.

Thaumastocera vittata Surcouf. Tabanidæ. Côte d'Ivoire. Ibid., p. 241.

Stigmatophthalmus Lutzi Surcouf. Tabanida. Santa-Fé-de-Bogota. Ibid., p. 242.

Buplex Bazini Surcouf. Tabanidæ. Kon-ling. Ibid., p. 243.

F. LARrousse.

\title{
Répertoire des hôtes nouveaux
}

depuis le $1^{\text {er }}$ Janvier 1923

\section{Hémiptères}

Triatoma brasiliensis. Reduvidæ. Rectum. Schizotrypanum Cruzi (Chagas, 1909). Rio Grande do Norte, Brésil. C. Pinto. Brazil-medico, XXXVII, 10 février 1923 , p. 73.

F. LARrousse.

Le Gérant: F. Amirault.

Cahors, Imprimerie CouesLant (personnel intéressé). - $27.28 j$ 